\title{
An inter-laboratory survey of paediatric bilirubin analyses
}

\author{
A. ST. JOHN' 1 AND L. A. PENBERTHY
}

From the Department of Clinical Biochemistry, Flinders Medical Centre, Bedford Park 5042, South Australia

SUMMARY A regional quality control trial of paediatric bilirubin analyses is described. The overall performance of the group was unsatisfactory with an unacceptably high inter-laboratory variation. The use of a common standard produced improvement in performance but it is concluded that the poor performance was also due to methodological problems. This lack of agreement between laboratories is a major problem when patients are transferred between hospitals.

Paediatric bilirubin analyses are essential in the management of neonates, especially if they become or remain jaundiced several days after birth. Clinical decisions whether to institute phototherapy or exchange transfusion are highly dependent upon the results of such analyses.

Most laboratories employ techniques for the analysis of paediatric specimens that are different from those used for adult patients and, while methodologies appropriate to the latter feature in most external quality control surveys such as the College of American Pathologists Survey and Wellcome Group Quality Control Programme, little attention has been devoted to inter-laboratory quality control trials for paediatric bilirubins. Accordingly, we have conducted an inter-laboratory quality control survey of this analysis within South Australia.

\section{Material and methods}

Table 1 shows the laboratories, the methods they used, and their methods of standardisation. Laboratories using two methods are entered twice, for example, A1, A2.

The methods used in the various laboratories were:

1 Differential spectroscopy by one of two methods

(a) dilution of plasma in phosphate buffer and measurement of the absorbance at 455 and 575 nm (White et al., 1958);

(b) dilution of plasma in borate buffer with measure-

1Present address: Department of Clinical Biochemistry, Royal Perth Hospital, Perth, Western Australia 6001.

Received for publication 12 February 1979
Table 1 Participating laboratories, methods, and standards

\begin{tabular}{lll}
\hline Laboratory code & Methods & Standards \\
\hline $\mathbf{A}_{\mathbf{1}}$ & Malloy-Evelyn & Commercial sera \\
$\mathbf{A}_{\mathbf{2}}$ & Bilirubinometer & Methyl orange \\
$\mathbf{B}$ & Jendrassik-Grof & Commercial sera \\
$\mathbf{C}_{\mathbf{1}}$ & Jendrassik-Grof & Commercial sera \\
$\mathbf{C}_{\mathbf{2}}$ & Bilirubinometer & Optical glass filter \\
$\mathbf{D}$ & Jendrassik-Grof & Commercial sera \\
$\mathbf{E}_{\mathbf{1}}$ & Malloy-Evelyn & Commercial sera \\
$\mathbf{E}_{\mathbf{2}}$ & Bilirubinometer & Methyl orange \\
$\mathbf{F}_{1}$ & Bilirubinometer & Optical glass filter \\
$\mathbf{F}_{\mathbf{2}}$ & Jendrassik-Grof & Commercial sera \\
$\mathbf{G}$ & Bilirubinometer & Methyl orange \\
$\mathbf{H}$ & Spectroscopy & Commercial sera \\
$\mathbf{I}$ & Spectroscopy & Commercial sera \\
$\mathbf{J}$ & Spectroscopy & Commercial sera \\
$\mathbf{K}$ & Bilirubinometer & Methyl orange \\
$\mathbf{L}$ & Spectroscopy & Commercial sera \\
$\mathbf{M}$ & Jendrassik-Grof & Commercial sera \\
\hline
\end{tabular}

ment of the absorbance at 466 and $522 \mathrm{~nm}$ (Hertz et al., 1974).

In both methods correction for haemolysis is achieved by measurement at the latter two wavelengths.

2 Bilirubinometers, of the Americal Optical Company (Buffalo, New York 14215) or the Advanced Instrument design (Needham Heights, Massachusetts 02194);

3 The diazotisation procedure of Jendrassik and Grof (1938);

4 The Malloy and Evelyn (1937) technique.

Specimens were distributed on six different occasions, all being prepared at a single distribution point. Six different types of commercial, lyophilised sera were used: Dade Bilirubin Control (Dade Division, American Hospital Supply Corporation, Miami, USA), Behringwerke Bilirubin Standard (Behringwerke AG, Hoechst Italia, Milan, Italy), 
Wellcomtrol III (Wellcome Reagent, Beckenham, UK), Hyland Elevated Bilirubin (Travenol Laboratories, Costa Mesa, California, USA), and Versatol Paediatric and Versatol A (General Diagnostics, Norris Plains, New Jersey, USA). Dade Bilirubin Control was distributed in duplicate on one occasion and in a single sample on a following distribution. All other sera detailed in Table 4 were distributed only once. All commercial sera were reconstituted according to the manufacturer's specifications, diluted further if required, and aliquoted accordingly. Diluted specimens were found to remain stable for 36 hours provided they were kept at $4{ }^{\circ} \mathrm{C}$ and protected from the light. Two types of human material were distributed: pooled adult plasma from jaundiced, hepatitis $B$ antigen negative subjects and post-exchange plasma from a neonate with $R h$ incompatibility.

All specimens were distributed in plastic tubes. Care was taken to protect them from the light during and after preparation. Laboratories were asked to store specimens at $4^{\circ}-8^{\circ} \mathrm{C}$ and to analyse them at the same time.

If laboratories were unable to analyse the specimens at the date and time requested they were asked to inform us accordingly so that we could exclude such results from the final calculations. In this way we minimised possible differences in results due to changes in the material distributed. In view of the considerable distance that some laboratories were from the distribution point the time of analysis was approximately 30-36 hours after preparation.

\section{Results and discussion}

Paediatric bilirubin analyses should be accurate and precise in view of their clinical importance. There are several biochemical criteria for clinical intervention when treating jaundiced neonates. One criterion is the rate of increase of the plasma bilirubin concentration. Swyer (1975) states that a bilirubin concentration increasing more than 8 $\mu \mathrm{mol} / 1(0.5 \mathrm{mg} / 100 \mathrm{ml}) / \mathrm{h}$ is an indication for exchange transfusion. The aim of the laboratory should, therefore, be to produce precise results that can confidently detect a change of concentration between two samples taken a few hours apart. Lathe (1970) has pointed out that difficulties in patient management may arise if too short a time interval between analyses is chosen when looking for significant changes in bilirubin concentration. However, it is our experience that such advice is largely unheeded, and when there is a possibility that an exchange transfusion may be required, clinicians commonly request analyses at too short time intervals.
Table 2 Results returned for Dade Bilirubin Control (stated value $364 \mu \mathrm{mol} / \mathrm{l}$ )

\begin{tabular}{lllll}
\hline Distribution & No. of labs & Mean & $S D$ & CV\% \\
\hline First & 15 & 361 & $30 \cdot 9$ & $8 \cdot 6$ \\
Second & 17 & 362 & $43 \cdot 5$ & $12 \cdot 0$ \\
Second & 17 & 366 & $42 \cdot 1$ & $11 \cdot 5$ \\
\hline
\end{tabular}

Table 2 shows the group results for Dade Bilirubin Control from two of the distributions. Although the group mean values for these three specimens are relatively constant and agree with the manufacturer's stated value, the intra-laboratory precision (Table 3 ) attained is unsatisfactory. Using the within-run standard deviation of $7.7 \mu \mathrm{mol} / 1$ a difference of $22 \mu \mathrm{mol} / \mathrm{l}(2.8 \times 7 \cdot 7)$ between results at the level of $364 \mu \mathrm{mol} / 1$ is necessary to give a $95 \%$ probability that two results are significantly different. In order to be certain that the plasma bilirubin was increasing at more than $8 \mu \mathrm{mol} / 1$ it would be necessary to perform analyses not less than 3 hours apart. However, in practice a difference of $78 \mu \mathrm{mol} / 1$ ( 2.8 times the day-to-day precision of $28.0 \mu \mathrm{mol} / \mathrm{l})$ reflects the average performance of laboratories in this clinical situation; that is, up to 10 hours between samples may be necessary confidently to identify a change in bilirubin concentration. This is unsatisfactory for patient management.

A second criterion for clinical intervention is the beginning of treatment when the plasma bilirubin concentration exceeds a defined level, depending upon the age of the neonate. Since an absolute figure in terms of bilirubin concentration is used by clinicians, it is essential that individual laboratories can produce accurate results for satisfactory patient management. Moreover, it is important that all laboratories in the group produce the same result, since neonates are often transferred from outlying hospitals to special-care hospitals when there is a possibility of phototherapy or exchange transfusions being required.

There is a lack of agreement between laboratories irrespective of whether we consider lyophilised controls or human pooled sera (Table 4). With this small group it is not possible to use the criterion that the mean value obtained by the group, excluding outliers, approximates the true value for that con-

Table 3 Intra-laboratory precision using Dade Bilirubin Control (stated value $364 \mu \mathrm{mol} / \mathrm{l}$ )

\begin{tabular}{llr}
\hline Precision & No. of labs & $S D$ \\
\hline Within-run & 17 & $7 \cdot 7$ \\
Day-to-day & 15 & $28 \cdot 0$ \\
\hline & & \\
Calculated using the difference between duplicates, ie, SD $=\sqrt{\frac{\Sigma \mathrm{d}}{2 \mathrm{n}}}$
\end{tabular}


Table 4 Summary of results returned for other sera distributed

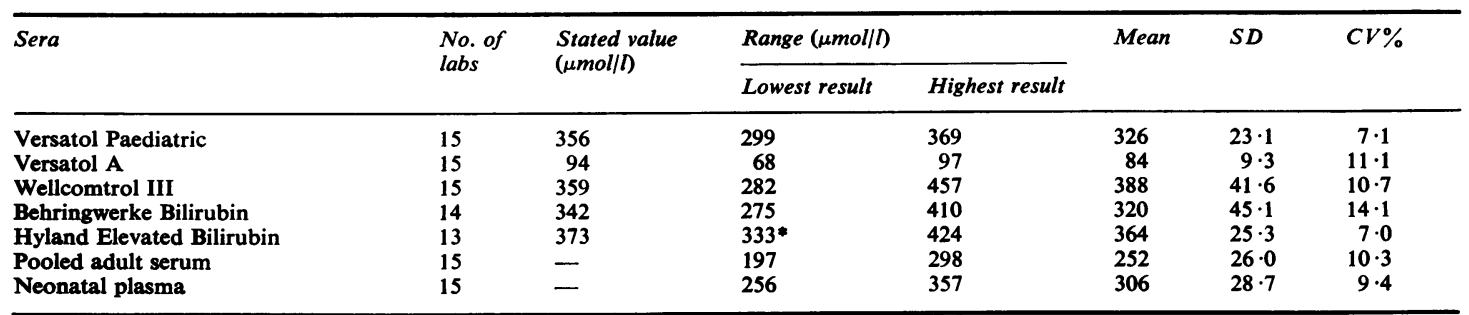

*Results of 142 and $270 \mu \mathrm{mol} / 1$ not included in calculations.

stituent (Whitehead et al., 1973). However, it is clear that the group performance in terms of accuracy does not meet clinical requirements.

Because of the variation in standards (Table 1) we investigated the effect on inter-laboratory variation when all laboratories used a common standard. All laboratories used a neonatal plasma, to which we had assigned a value approximating its true bilirubin concentration as an alternative to their normal standard material. In Table 5 we compare the values obtained from laboratories for commercial and human sera using their own and our standard. The mean values for the human sera are not significantly different $(P>0.5)$ whereas the means for commercial lyophilised sera differ significantly $(0.01<P<0.05)$. Furthermore, when the common standard was used the inter-laboratory precision improved significantly $(P<0.05)$ in both instances. This improvement in performance could be expected when all laboratories used a common standard. However, it does reinforce the need for laboratories in a common geographical area to agree on the use of such a standard.

Table 5 Effect on precision when using a common standard

\begin{tabular}{|c|c|c|c|c|}
\hline & \multicolumn{2}{|c|}{ Commercial lyophilised sera } & \multicolumn{2}{|c|}{ Human sera } \\
\hline & $\begin{array}{l}\text { Individual } \\
\text { lab std }\end{array}$ & $\begin{array}{l}\text { Common } \\
\text { std }\end{array}$ & $\begin{array}{l}\text { Individual } \\
\text { lab std }\end{array}$ & $\begin{array}{l}\text { Common } \\
\text { std }\end{array}$ \\
\hline $\begin{array}{l}\text { Mean } \\
\text { SD } \\
\text { CV\% }\end{array}$ & $\begin{array}{l}279 \\
29 \cdot 0 \\
10 \cdot 4\end{array}$ & $\begin{array}{r}299 \\
15 \cdot 8 \\
5 \cdot 3\end{array}$ & $\begin{array}{r}203 \\
14 \cdot 4 \\
7 \cdot 1\end{array}$ & $\begin{array}{r}215 \\
7 \cdot 9 \\
3 \cdot 7\end{array}$ \\
\hline CV\% & \multicolumn{2}{|c|}{$n=15$} & \multicolumn{2}{|c|}{$\mathrm{n}=12$} \\
\hline
\end{tabular}

Table 6 Overall precision of methods used

\begin{tabular}{lcl}
\hline Method & No. of results & Average SD \\
\hline Spectroscopy & 76 & $17 \cdot 5$ \\
Jendrassik-Grof & 103 & $21 \cdot 6$ \\
Bilirubinometer & 108 & $23 \cdot 1$ \\
All methods & 322 & $27 \cdot 7$ \\
\hline
\end{tabular}

Table 6 summarises the overall precision of the three main analytical methods. By analysing the individual variances we have calculated a mean variance and subsequently an average standard deviation for each method. Spectroscopy methods are significantly more precise $(0.05>\mathrm{P}>0.01)$ than Jendrassik and Grof methods. The Michaëlsson et al. (1965) modification of Jendrassik and Grof has been recommended as the method of choice for bilirubin assay by some workers (Billing et al., 1971; Doumas et al., 1973). In a paediatric laboratory, where one is dealing with very small samples, diazo methods such as Jendrassik and Grof, or Malloy and Evelyn, involve a number of manipulations with very small volumes and thus require considerable expertise. Spectroscopy techniques require only a preliminary dilution step and therefore allow for fewer potential errors to occur. This is reflected by the marked improvement in precision, compared to diazo procedures, when using these simple methods.

In this laboratory, each of 19 'on-call' personnel was asked to measure the bilirubin concentration of a neonatal plasma using both a bilirubinometer and a Jendrassik and Grof technique. The mean values obtained were 239 and $241 \mu \mathrm{mol} / \mathrm{l}$ respectively. However the bilirubinometer results gave a coefficient of variation of $1.3 \%$ compared with $5.1 \%$ for the Jendrassik and Grof method. Attention has recently been drawn by Ebbesen (1977) to the method simplicity of spectrophotometry. It is also noted that a one-step modification of the Malloy-Evelyn procedure has recently been proposed by Shihabi and Scaro (1977). The suggested advantage of diazo procedures such as Michaëlsson's over spectroscopy has been their ability to overcome interferences. However, extensive work by Hertz et al. (1974) into various formulae used for calculating bilirubin concentrations by direct spectroscopy has shown that carefully derived formulae can compensate for interferences from haemoglobin, methaemalbumin, and turbidity, producing good agreement between this method and diazo procedure.

We are investigating further the value of method 
simplification with emphasis on the use of appropriate standards for spectrophotometric methods and bilirubinometers since these methods offer the precision required with the small volumes of sample and reagent necessary for neonatal analyses. However, bilirubinometers and spectroscopy measure only total bilirubin and, in order to estimate the concentration of bilirubin esters, it is necessary further to investigate simple methods for such estimations.

It may be significant that no laboratory was using prepared bilirubin standards, as has been recommended by a number of groups (Joint Committee Report, 1962; Billing et al., 1971). Such standards are difficult to prepare, however, and the work involved would probably be beyond the facilities of our smaller laboratories.

This work was performed as part of the Quality Control Programme organised by the South Australian Branch of the Australian Association of Clinical Biochemists. We thank all the laboratories that participated in the survey.

\section{References}

Billing, B., Haslam, R., and Wald, N. (1971). Bilirubin standards and the determination of bilirubin by manual and Technicon Autoanalyser methods. Annals of Clinical Biochemistry, 8, 21-30.

Doumas, B. T., Perry, B. W., Sasse, E. A., and Straumfjord, J. V., Jr. (1973). Standardisation in bilirubin assays: evaluation of selected methods and stability of bilirubin solutions. Clinical Chemistry, 19, 984-993.

Ebbesen, F. (1977). Determination of serum bilirubin concentration during phototherapy of newborns and in vitro: results compared by the direct spectrometric method and the diazo method. Clinical Chemistry, 23, 695-699.

Hertz, H., Dybkaer, R., and Lauritzen, M. (1974). Direct spectrometric determination of the concentration of bilirubins in serum. Scandinavian Journal of Clinical and Laboratory Investigation, 33, 215-230.

Jendrassik, L., and Grof, P. (1938). Vereinfachte photometrische Methoden zur Bestimmung des Bilirubins. Biochemische Zeitschrift, 297, 81-89.

Joint Committee Report (1962). Recommendation on a uniform bilirubin standard. Clinical Chemistry, 8, 405-407.

Lathe, G. H. (1970). Jaundice of the newborn infant. Annals of Clinical Biochemistry, 7, 81-85.

Malloy, H. T., and Evelyn, K. A. (1937). The determination of bilirubin with the photoelectric colorimeter. Journal of Biological Chemistry, 119, 481-490.

Michaëlsson, M., Nosslin, B., and Sjölin, S. (1965). Plasma bilirubin determination in the newborn infant. Pediatrics, 35, 925-931.

Shihabi, Z. K., and Scaro, J. (1977). A modified MalloyEvelyn procedure for total bilirubin in microsamples. American Journal of Medical Technology, 43, 1004-1007.

Swyer, P. R. (1975). The Intensive Care of the Newly Born (Monographs in Paediatrics 6). Karger, Basle.

White, D., Haidar, G. A., and Reinhold, J. G. (1958). Spectrophotometric measurement of bilirubin concentrations in the serum of the newborn by the use of a microcapillary method. Clinical Chemistry, 4, 211-222.

Whitehead, T. P., Browning, D. M., and Gregory, A. A. (1973). A comparative survey of the results of analyses of blood serum in clinical chemistry laboratories in the United Kingdom. Journal of Clinical Pathology, 26, $435-445$.

Requests for reprints to: L. A. Penberthy, Flinders Medical Centre, Bedford Park, South Australia 5042. 